\title{
On the Creation of a Stable Drop-Like Static Meniscus, Appropriate for the Growth of a Single Crystal Tube with Prior Specified Inner and Outer Radii
}

\author{
Stefan Balint ${ }^{\mathbf{1}}$ and Agneta M. Balint ${ }^{\mathbf{2}}$ \\ ${ }^{1}$ Faculty of Mathematics and Computer Science, West University of Timisoara, Bulv. V.Parvan 4, \\ 300223 Timisoara, Romania \\ ${ }^{2}$ Faculty of Physics, West University of Timisoara, Bulv. V.Parvan 4, \\ 300223 Timisoara, Romania
}

Correspondence should be addressed to Agneta M. Balint, agneta.balint@gmail.com

Received 22 February 2009; Revised 6 May 2009; Accepted 24 May 2009

Recommended by Ben T. Nohara

\begin{abstract}
A theoretical procedure for the creation of a stable drop-like static meniscus, appropriate for the growth of a single crystal tube, with a priori specified inner and outer radius, is presented. The method locates the controllable part $p$ of the pressure difference across the free surface. It consists in a set of calculus, which leads to the determination of the melt column height (between the horizontal crucible melt level and the shaper top level) in function of the pressure of the gas flow (introduced in the furnace for release the heat) in order to obtain the desired meniscus. The procedure is presented in general and is numerically illustrated for InSb tubes. The novelty is the algorithm for the exact determination of $p$, which has to be used, the determination of the melt column height, and the evaluation of the effect of shaper radii. The setting of the thermal conditions, which assure that for the obtained static meniscus the solidification conditions are satisfied at the "right" places, is not considered here.
\end{abstract}

Copyright (C) 2009 S. Balint and A. M. Balint. This is an open access article distributed under the Creative Commons Attribution License, which permits unrestricted use, distribution, and reproduction in any medium, provided the original work is properly cited.

\section{Introduction}

The conventional melt growth techniques, as Bridgman growth [1-3] or Czochralski pulling [4-6] of single crystals, typically produce ingots of circular or square cross-sections which need to be cut in hundreds of slices to produce wafers. Using these processes, it is difficult to produce thin wafers from an ingot without wasting $40 \%-50 \%$ of material as kerfs during the cutting process. For this reason the E.F.G. technology can be more appropriate to produce single crystals with prescribed shapes and sizes which can be used without additional machining. 
The growth of silicon tubes by E.F.G. process was first reported by Erris et al. [7]. In [7] a theory of tube growth by E.F.G. process is developed to show the dependence of tube wall thickness on the growth variables. The theory uses approximation reported in $[8,9]$, and it has been shown to be a useful tool understanding the feasible limits of the wall thickness control. A more accurate predictive model would require an increase of the acceptable tolerance range introduced by approximation.

Later, the heat flow in a tube growth system was analyzed in [10-19].

The state of the arts at the time 1993-1994, concerning the calculation of the meniscus shape in general in the case of the growth by E.F.G. method is summarized in [20]. According to [20], for the general differential equation describing the free surface of a liquid meniscus, possessing axial symmetry, there are no complete analysis and solution. For the general equation only numerical integrations were carried out for a number of process parameter values that were of practical interest at the moment. The authors of [21,22] consider automated crystal growth processes based on weight sensors and computers. They give an expression for the weight of the meniscus, contacted with crystal and shaper of arbitrary shape, in which there are two terms related to the hydrodynamic factor.

In [23] it is shown that the hydrodynamic factor is too small to be considered in the automated crystal growth. In [24] a theoretical and numerical study of meniscus dynamics, under symmetric and asymmetric configurations, is presented. A meniscus dynamics model is developed to consider meniscus shape and its dynamics, heat and mass transfer around the die top and meniscus. Analysis reveals the correlations among tube thickness, effective melt height, pull rate, die top temperature, and crystal environmental temperature.

In [25] the effect of the controllable part of the pressure difference on the free surface shape of the static meniscus is analyzed for the tube growth by E.F.G. method for materials for which $0<\alpha_{c}<\pi / 2 ; 0<\alpha_{g}<\pi / 2 ; \alpha_{c}>\pi / 2-\alpha_{g}$.

The present paper concerns also the shape and the stability of the free surface of a static meniscus (pulling rate equal to zero). More precisely, it is shown in which kind the explicit formulas reported in [25] can be combined in order to create a stable static meniscus having a free surface with prescribed size and shape, which is appropriate for the growth of a single crystal tube having a priori specified inner and outer radii. The free surface of a static meniscus is appropriate for the growth of a single crystal tube of constant inner radius $r_{i}$ and constant outer radius $r_{e}$ if the angle between the tangent lines to the free surface at the points $\left(r_{i}, z_{i}\left(r_{i}\right)\right),\left(r_{e}, z_{e}\left(r_{e}\right)\right)$ (Figure 1 where the solidification conditions have to be assured) and the vertical is equal to the growth angle $\alpha_{g}$. Moreover, the function describing the free surface has to minimize the energy functional of the melt column (i.e., the meniscus has to be stable). In this paper we give a procedure for the choice of the melt column height, between the horizontal crucible melt level and shaper top level and of the pressure of the gas flow introduced in the furnace (for release the heat), in order to create a static meniscus of which free surface is appropriate for the growth of a single crystal tube of constant inner radius $r_{i}$ and outer radius $r_{e}$. The thermal problem concerning the setting of the thermal conditions, which assure that for the obtained static meniscus at the level $z_{i}\left(r_{i}\right), z_{e}\left(r_{e}\right)$ the solidification conditions are satisfied is not considered in this paper. The novelty consists in the fact that the free surface is not approximated by an arc with constant curvature, the computation takes into account the pressure of the gas flow, and the stability of the free surface is assured. 


\section{The Free Surfaces Equations and the Pressure Difference Limits}

For a single crystal tube growth by E.F.G. technique, in hydrostatic approximation, the outer free surface equation of the static meniscus is

$$
z_{e}^{\prime \prime}=\frac{\rho \cdot g \cdot z_{e}-p_{e}}{r}\left[1+\left(z_{e}^{\prime}\right)^{2}\right]^{3 / 2}-\frac{1}{r} \cdot\left[1+\left(z_{e}^{\prime}\right)^{2}\right] \cdot z_{e}^{\prime} ; \quad r \in\left[\frac{R_{g i}+R_{g e}}{2}, R_{g e}\right]
$$

and the inner free surface equation is

$$
z_{i}^{\prime \prime}=\frac{\rho \cdot g \cdot z_{i}-p_{i}}{\gamma}\left[1+\left(z_{i}^{\prime}\right)^{2}\right]^{3 / 2}-\frac{1}{r} \cdot\left[1+\left(z_{i}^{\prime}\right)^{2}\right] \cdot z_{i}^{\prime} ; \quad r \in\left[R_{g i} \frac{R_{g i}+R_{g e}}{2}\right]
$$

Here, $\gamma$ is the surface tension of the melt; $\rho$ is the melt density; $g$ is the gravitational acceleration; $z_{e}, z_{i}$ are the coordinates with respect to the $\mathrm{Oz}$ axis, directed vertically upwards; $r$ is the radial coordinate with respect to the $\mathrm{Or}$ axis, oriented horizontal; $R_{g e}, R_{g i}$ are the outer and inner radius of the shaper, respectively; $p_{e}, p_{i}$ are the pressure difference across the outer and inner free surface, respectively:

$$
p_{e}=p_{m}-p_{g}^{e}-\rho \cdot g \cdot H, \quad p_{i}=p_{m}-p_{g}^{i}-\rho \cdot g \cdot H
$$

In (2.3), $p_{m}$ denotes the hydrodynamic pressure in the meniscus melt due to the thermal and Marangoni convection; $p_{g}^{e}, p_{g}^{i}$ are the pressure of the gas flow introduced in the exterior and in the interior of the tube, respectively, for releasing the heat from the inner and outer side of the tube wall; $H$ denotes the melt column "height" between the horizontal crucible melt level, and the shaper top level (Figure 1). $H$ is positive when the crucible melt level is under the shaper top level and it is negative when the shaper top level is under the crucible melt level.

The solution $z_{e}=z_{e}(r)$ of (2.1) has to satisfy the following conditions:

$$
\begin{gathered}
z_{e}^{\prime}\left(r_{e}\right)=-\tan \left(\frac{\pi}{2}-\alpha_{g}\right), \\
z_{e}^{\prime}\left(R_{g e}\right)=-\tan \alpha_{c} \\
z_{e}\left(R_{g e}\right)=0 \text { and } z_{e}(r) \text { is strictly decreasing on }\left[r_{e}, R_{g e}\right],
\end{gathered}
$$

where $r_{e} \in\left(\left(R_{g i}+R_{g e}\right) / 2, R_{g e}\right)$ is the tube outer radius; $\alpha_{g}$ is the growth angle; $\alpha_{c}$ is the contact angle between the outer free surface and the outer edge of the shaper top and $0<$ $\alpha_{c}<\pi / 2 ; 0<\alpha_{g}<\pi / 2 ; \pi / 2-\alpha_{g}<\alpha_{c}$ (Figure 1 ).

Condition (2.4a) expresses that at the point $\left(r_{e}, z_{e}\left(r_{e}\right)\right)$ (the left end of the outer free surface), where the solidification has to be realized, the angle between the tangent line to the free surface and the vertical is equal to the growth angle $\alpha_{g}$ (i.e., the tangent to the tube outer wall is vertical).

Condition $(2.4 \mathrm{~b})$ expresses that at the point $\left(R_{g e}, 0\right)$ (the right end of the outer free surface, where the free surface is attached to the shaper edge), the angle between the tangent line to the free surface and horizontal (i.e., the contact angle) is equal to $\alpha_{c}$. 


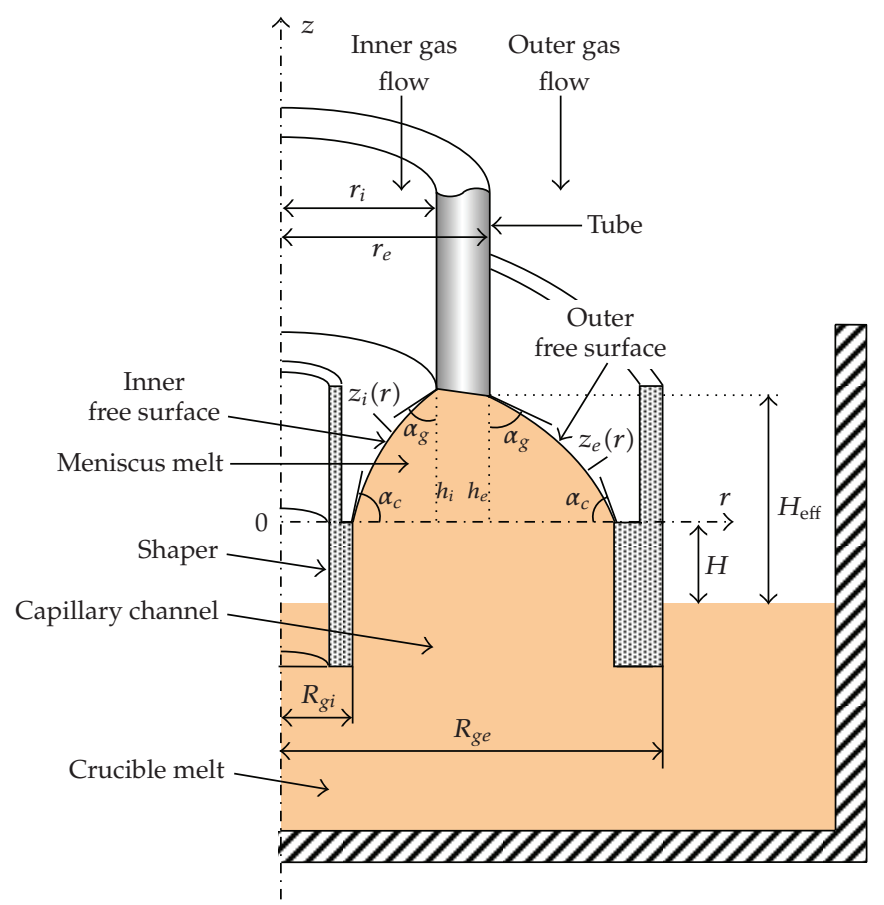

Figure 1: Axisymmetric meniscus geometry in the tube growth by E.F.G. method.

Condition (2.4c) expresses that at the point $\left(R_{g e}, 0\right)$ the free surface is attached to the shaper edge. column:

Moreover, the solution $z_{e}=z_{e}(r)$ has to minimize the energy functional of the melt

$$
\begin{gathered}
I_{e}(z)=\int_{r_{e}}^{R_{g e}}\left\{r \cdot\left[1+\left(z^{\prime}\right)^{2}\right]^{1 / 2}+\frac{1}{2} \cdot \rho \cdot g \cdot z^{2}-p_{e} \cdot z\right\} \cdot r \cdot d r \\
z\left(r_{e}\right)=h_{e}>0, \quad z\left(R_{g e}\right)=0 .
\end{gathered}
$$

The solution $z_{i}=z_{i}(r)$ of (2.2) has to satisfy the following conditions:

$$
\begin{gathered}
z_{i}^{\prime}\left(R_{g i}\right)=\tan \alpha_{c}, \\
z_{i}^{\prime}\left(r_{i}\right)=\tan \left(\frac{\pi}{2}-\alpha_{g}\right), \\
z_{i}\left(R_{g i}\right)=0 \text { and } z_{i}(r) \text { is strictly increasing on }\left[R_{g i}, r_{i}\right],
\end{gathered}
$$

where: $r_{i} \in\left(R_{g i}\left(R_{g i}+R_{g e}\right) / 2\right)$ is the tube inner radius; $\alpha_{g}$ is the growth angle; $\alpha_{c}$ is the contact angle between the inner free surface and the inner edge of the shaper top and $0<$ $\alpha_{c}<\pi / 2 ; 0<\alpha_{g}<\pi / 2 ; \pi / 2-\alpha_{g}<\alpha_{c}$ (Figure 1 ). 
Condition (2.6a) expresses that at the point $\left(R_{g i}, 0\right)$ (the left end of the inner free surface, where the free surface is attached to the shaper edge), the angle between the tangent line to the free surface and horizontal (i.e., the contact angle) is equal to $\alpha_{c}$.

Condition (2.6b) expresses that at the point $\left(r_{i}, z_{i}\left(r_{i}\right)\right)$ (the right end of the inner free surface), where the solidification has to be realized, the angle between the tangent line to the free surface and the vertical is equal to the growth angle $\alpha_{g}$ (i.e., the tangent to the tube inner wall is vertical).

Condition (2.6c) expresses that at the point $\left(R_{g i}, 0\right)$ the free surface is attached to the shaper edge.

Moreover, the solution $z_{i}=z_{i}(r)$ has to minimize the energy functional of the melt column:

$$
\begin{gathered}
I_{i}(z)=\int_{R_{g i}}^{r_{i}}\left\{r \cdot\left[1+\left(z^{\prime}\right)^{2}\right]^{1 / 2}+\frac{1}{2} \cdot \rho \cdot g \cdot z^{2}-p_{i} \cdot z\right\} \cdot r \cdot d r, \\
z\left(R_{g i}\right)=0, \quad z\left(r_{i}\right)=h_{i}>0 .
\end{gathered}
$$

Based on the mathematical theorems, rigorously proven in [25], the following statements, regarding the creation of an appropriate meniscus, can be formulated.

\section{Statement 1}

If the solution of the initial value problem (IVP)

$$
\begin{gathered}
z_{e}^{\prime \prime}=\frac{\rho \cdot g \cdot z_{e}-p_{e}}{r}\left[1+\left(z_{e}^{\prime}\right)^{2}\right]^{3 / 2}-\frac{1}{r} \cdot\left[1+\left(z_{e}^{\prime}\right)^{2}\right] \cdot z_{e}^{\prime} \\
z_{e}\left(R_{g e}\right)=0, \quad z_{e}^{\prime}\left(R_{g e}\right)=-\tan \alpha_{c}
\end{gathered}
$$

is convex, then it does not represent the outer free surface of an appropriate drop-like static meniscus.

Comment 1. The above statement shows that the solutions of IVP (2.8), which represent the outer free surface of an appropriate drop-like static meniscus, can be obtained only for those values of $p_{e}$ for which the solution is not globally convex.

\section{Statement 2}

If $p_{e}<\left(\gamma / R_{g e}\right) \cdot \sin \alpha_{c}$, then the solution of the IVP (2.8) is globally convex, and it does not represent the outer free surface of an appropriate drop-like static meniscus.

Comment 2. The above statement locates a set of $p_{e}$ values which are not appropriate for the creation of a drop-like meniscus. 


\section{Statement 3}

If the solution $z_{e}=z_{e}(r)$ of the nonlinear boundary value problem (NLBVP) (2.1), (2.4a), $(2.4 \mathrm{~b})$, and (2.4c) represents the outer free surface of an appropriate concave static meniscus on the closed interval $\left[R_{g e} / n, R_{g e}\right]$, with $1<n<\left(R_{g i}+R_{g e}\right) / 2$, then the following inequalities hold:

$$
\begin{aligned}
& \frac{n}{n-1} \cdot \gamma \cdot \frac{\alpha_{c}+\alpha_{g}-\pi / 2}{R_{g e}} \cdot \cos \alpha_{c}+\frac{\gamma}{R_{g e}} \cdot \cos \alpha_{g} \\
& \quad \leq p_{e} \leq \frac{n}{n-1} \cdot \gamma \cdot \frac{\alpha_{c}+\alpha_{g}-\pi / 2}{R_{g e}} \cdot \sin \alpha_{g}+\frac{n-1}{n} \cdot \rho \cdot g \cdot R_{g e} \cdot \tan \alpha_{c}+n \cdot \frac{\gamma}{R_{g e}} \cdot \sin \alpha_{c} .
\end{aligned}
$$

Comment 3. The above statement shows that in order to obtain the outer free surface of a concave static meniscus, appropriate for the growth of a tube of outer radius $r_{e}=R_{g e} / n, p_{e}$ has to be searched in the range defined by the inequalities (2.9).

\section{Consequence 1}

If $n=2 \cdot R_{g e} /\left(R_{g i}+R_{g e}\right)\left(r_{e}\right.$ is the middle point of the interval $\left.\left[R_{g i}, R_{g e}\right]\right)$, then $r_{e}=R_{g e} / n=$ $\left(R_{g i}+R_{g e}\right) / 2$ and inequalities (2.9) become

$$
\begin{aligned}
2 \cdot \gamma \cdot \frac{\alpha_{c}+\alpha_{g}-\pi / 2}{R_{g e}-R_{g i}} \cdot \cos \alpha_{c}+\frac{\gamma}{R_{g e}} \cdot \cos \alpha_{g} \\
\quad \leq p_{e} \leq 2 \cdot \gamma \cdot \frac{\alpha_{c}+\alpha_{g}-\pi / 2}{R_{g e}-R_{g i}} \cdot \sin \alpha_{g}+\frac{\rho \cdot g \cdot\left(R_{g e}-R_{g i}\right)}{2} \cdot \tan \alpha_{c}+\frac{2 \cdot \gamma}{R_{g e}+R_{g i}} \cdot \sin \alpha_{c} .
\end{aligned}
$$

Comment 4. The above consequence shows that in order to obtain the outer free surface of a concave static meniscus, appropriate for the growth of a tube of outer radius $r_{e}=\left(R_{g i}+\right.$ $\left.R_{g e}\right) / 2, p_{e}$ has to be searched in the range defined by the inequalities (2.10).

\section{Consequence 2}

If $p_{e}$ verifies the inequality

$$
p_{e}<2 \cdot \gamma \cdot \frac{\alpha_{c}+\alpha_{g}-\pi / 2}{R_{g e}-R_{g i}} \cdot \cos \alpha_{c}+\frac{\gamma}{R_{g e}} \cdot \cos \alpha_{g}
$$

then there is no $r_{e}$ in the closed interval $\left[\left(R_{g i}+R_{g e}\right) / 2, R_{g e}\right]$ for which the NLBVP (2.1), (2.4a), $(2.4 \mathrm{~b})$, and $(2.4 \mathrm{c})$ possesses a concave solution.

Comment 5. The above consequence shows that if $p_{e}$ is in the range defined by the inequality (2.11), then it is impossible to obtain a static meniscus having concave outer free surface which is appropriate for the growth of a tube of outer radius $r_{e}$ situated in the range $\left[\left(R_{g i}+\right.\right.$ $\left.\left.R_{g e}\right) / 2, R_{g e}\right]$. 
Consequence 3

If $n \rightarrow 1$, then $r_{e}=R_{g e} / n \rightarrow R_{g e}$ and, according to (2.9), $p_{e} \rightarrow+\infty$.

Comment 6. The above consequence shows that in order to obtain the outer free surface of a concave static meniscus, appropriate for the growth of a tube of outer radius $r_{e} \approx R_{g e}, p_{e}$ has to be very high.

\section{Statement 4}

If $n$ and $p_{e}$ verify the inequalities

$$
\begin{gathered}
1<n<\frac{2 \cdot R_{g e}}{R_{g e}+R_{g i}}, \\
p_{e}>\frac{n}{n-1} \cdot \gamma \cdot \frac{\alpha_{c}+\alpha_{g}-\pi / 2}{R_{g e}} \cdot \sin \alpha_{g}+\frac{n-1}{n} \cdot \rho \cdot g \cdot R_{g e} \cdot \tan \alpha_{c}+n \cdot \frac{\gamma}{R_{g e}} \cdot \sin \alpha_{c}
\end{gathered}
$$

then there exist $r_{e} \in\left[R_{g e} / n, R_{g e}\right]$ and a concave solution of the NLBVP (2.1), (2.4a), (2.4b), and $(2.4 \mathrm{c})$ on the interval $\left[r_{e}, R_{g e}\right]$.

Comment 7. The above consequence shows that if $n$ and $p_{e}$ verify (2.12), then a static meniscus having concave outer free surface, appropriate for the growth of a tube of outer radius $r_{e}$ situated in the range $\left[R_{g e} / n, R_{g e}\right]$, is obtained.

\section{Consequence 4}

If for $1<n^{\prime}<n<2 \cdot R_{g e} /\left(R_{g i}+R_{g e}\right)$ and $p_{e}$ the following inequalities hold:

$$
\begin{gathered}
\frac{n}{n-1} \cdot \gamma \cdot \frac{\alpha_{c}+\alpha_{g}-\pi / 2}{R_{g e}} \cdot \sin \alpha_{g}+\frac{n-1}{n} \cdot \rho \cdot g \cdot R_{g e} \cdot \tan \alpha_{c}+n \cdot \frac{\gamma}{R_{g e}} \cdot \sin \alpha_{c} \\
<p_{e}<\frac{n^{\prime}}{n^{\prime}-1} \cdot \gamma \cdot \frac{\alpha_{c}+\alpha_{g}-\pi / 2}{R_{g e}} \cdot \cos \alpha_{c}+\frac{\gamma}{R_{g e}} \cdot \cos \alpha_{g}
\end{gathered}
$$

then there exist $r_{e}$ in the interval $\left[R_{g e} / n, R_{g e} / n^{\prime}\right]$ and a concave solution of the NLBVP (2.1), (2.4a), (2.4b), and (2.4c) on the interval $\left[r_{e}, R_{g e}\right]$.

Comment 8 . The above consequence shows that if $p_{e}$ is in the range defined by the inequalities (2.13), then a static meniscus having concave outer free surface, appropriate for the growth of a tube of outer radius $r_{e}$ situated in the range $\left[R_{g e} / n, R_{g e} / n^{\prime}\right]$, is obtained.

\section{Statement 5}

A concave solution $z_{e}=z_{e}(r)$ of the NLBVP (2.1), (2.4a), (2.4b), and (2.4c) is a weak minimum of the energy functional of the melt column. 
Comment 9. The above consequence shows that a static meniscus having a concave outer free surface, appropriate for the growth of a tube of outer radius $r_{e}$ situated in the range $\left[\left(R_{g i}+R_{g e}\right) / 2, R_{g e}\right]$, is stable.

\section{Statement 6}

If $n$ and $p_{e}$ satisfy the inequalities

$$
1<n<\frac{2 \cdot R_{g e}}{R_{g e}+R_{g i}}, \quad p_{e}>\frac{n-1}{n} \cdot g \cdot \rho \cdot R_{g e} \cdot \tan \alpha_{c}+n \cdot \frac{\gamma}{R_{g e}}
$$

then the solution $z_{e}=z_{e}(r)$ of the IVP (2.8) is concave on the interval $I \cap\left[R_{g e} / n, R_{g e}\right]$ where $I$ is the maximal interval of the existence of $z_{e}(r)$.

Comment 10. The above consequence shows that if $p_{e}$ is in the range defined by the inequalities (2.14), then eventually it can be used for the creation of a drop-like static meniscus, appropriate for the growth of a tube of outer radius $r_{e}$ in the range $\left[R_{g e} / n, R_{g e}\right]$.

\section{Statement 7}

If $p_{e}>\left(\gamma / R_{g e}\right) \cdot \sin \alpha_{c}$ and for a value $r_{e}=R_{g e} / n$, which satisfies $r_{e} \in\left(\left(R_{g i}+R_{g e}\right) / 2, R_{g e}\right)$, a static meniscus appropriate for the growth of a tube of outer radius $r_{e}$ exists, then for $p_{e}$ the following inequalities hold:

$$
\frac{\gamma}{R_{g e}} \cdot \sin \alpha_{c}<p_{e}<\rho \cdot g \cdot \frac{n-1}{n} \cdot R_{g e} \cdot \tan \alpha_{c}+n \cdot \frac{\gamma}{R_{g e}} \cdot \cos \alpha_{c}
$$

Comment 11. The above statement locates those values of $p_{e}$ for which eventually nonglobally concave (drop-like) static meniscus, appropriate for the growth of a tube of outer radius $r_{e}=R_{g e} / n$, exists.

\section{Statement 8}

If the solution of the IVP

$$
\begin{gathered}
z_{i}^{\prime \prime}=\frac{\rho \cdot g \cdot z_{i}-p_{i}}{r}\left[1+\left(z_{i}^{\prime}\right)^{2}\right]^{3 / 2}-\frac{1}{r} \cdot\left[1+\left(z_{i}^{\prime}\right)^{2}\right] \cdot z_{i}^{\prime} \\
z_{i}\left(R_{g i}\right)=0, \quad z_{i}^{\prime}\left(R_{g i}\right)=\tan \alpha_{c}
\end{gathered}
$$

is convex, then it does not represent the inner free surface of an appropriate drop-like static meniscus.

Comment 12. The above statement shows that the inner free surface of an appropriate droplike static meniscus can be obtained only for those values of $p_{i}$ for which the IVP (2.16) is not globally convex. 


\section{Statement 9}

If $p_{i}$ satisfies the inequality

$$
p_{i}<-\frac{\gamma}{R_{g i}}
$$

then the solution $z_{i}=z_{i}(r)$ of the IVP (2.15) is convex on the interval $I \cap\left[R_{g i},\left(R_{g i}+R_{g e}\right) / 2\right]$, where $I$ is the maximal interval of the existence of $z_{i}(r)$.

Comment 13. The above statement shows that if $p_{i}$ is in the range defined by the inequality (2.17), then it is impossible to obtain a static meniscus, appropriate for the growth of a tube of inner radius $r_{i}$ situated in the range $\left[R_{g i},\left(R_{g i}+R_{g e}\right) / 2\right]$.

\section{Statement 10}

If the solution $z_{i}=z_{i}(r)$ of the NLBVP (2.2) and (2.5) represents the inner free surface of an appropriate concave static meniscus on the closed interval $\left[R_{g i}, m \cdot R_{g i}\right]$ with $1<m<$ $\left(R_{g i}+R_{g e}\right) / 2 \cdot R_{g i}$, then the following inequalities hold:

$$
\begin{aligned}
& \frac{1}{m-1} \gamma \cdot \frac{\alpha_{c}+\alpha_{g}-\pi / 2}{R_{g i}} \cdot \cos \alpha_{c}-\frac{\gamma}{R_{g i}} \cdot \sin \alpha_{c} \leq p_{i} \\
& \quad \leq \frac{1}{(m-1)} \gamma \cdot \frac{\alpha_{c}+\alpha_{g}-\pi / 2}{R_{g i}} \cdot \sin \alpha_{g}+(m-1) \cdot \rho \cdot g \cdot R_{g i} \cdot \tan \alpha_{c}-\frac{\gamma}{m \cdot R_{g i}} \cdot \cos \alpha_{g} .
\end{aligned}
$$

Comment 14. The above statement shows that in order to obtain the inner free surface of a concave static meniscus, appropriate for the growth of a tube of inner radius $r_{i}=m \cdot R_{g i}, p_{i}$ has to be searched in the range defined by the inequalities (2.18).

\section{Consequence 5}

If $m=\left(R_{g i}+R_{g e}\right) / 2 \cdot R_{g i}$, then $r_{i}=\left(R_{g i}+R_{g e}\right) / 2$ (i.e., $r_{i}$ is the middle point of the interval $\left.\left\lfloor R_{g i}, R_{g e}\right\rfloor\right)$ and the inequalities (2.18) become:

$$
\begin{aligned}
2 \cdot \gamma \cdot \frac{\alpha_{c}+\alpha_{g}-\pi / 2}{R_{g e}-R_{g i}} \cdot \cos \alpha_{c}-\frac{\gamma}{R_{g i}} \cdot \sin \alpha_{c} \leq p_{i} \\
\quad \leq 2 \cdot \gamma \cdot \frac{\alpha_{c}+\alpha_{g}-\pi / 2}{R_{g e}-R_{g i}} \cdot \sin \alpha_{g}+\frac{\rho \cdot g \cdot\left[R_{g e}-R_{g i}\right]}{2} \cdot \tan \alpha_{c}-\frac{2 \cdot \gamma}{R_{g e}+R_{g i}} \cdot \cos \alpha_{g} .
\end{aligned}
$$

Comment 15. The above consequence shows that in order to obtain the inner free surface of a concave static meniscus, appropriate for the growth of a tube of inner radius $r_{i}=\left(R_{g i}+R_{g e}\right) / 2$, $p_{i}$ has to be searched in the range defined by the inequalities (2.19). 


\section{Consequence 6}

If $p_{i}$ verifies:

$$
p_{i}<2 \cdot \gamma \cdot \frac{\alpha_{c}+\alpha_{g}-\pi / 2}{R_{g e}-R_{g i}} \cdot \cos \alpha_{c}-\frac{\gamma}{R_{g i}} \cdot \sin \alpha_{c}
$$

then there is no $r_{i}$ in the closed interval $\left[R_{g i},\left(R_{g i}+R_{g e}\right) / 2\right]$ for which the NLBVP (2.2) and (2.5) possesses a concave solution.

Comment 16. The above consequence shows that if $p_{i}$ is in the range defined by the inequalities (2.19), then it is impossible to obtain a static meniscus, having concave inner free surface and appropriate for the growth of a tube of inner radius $r_{i}$ situated in the range $\left[R_{g i},\left(\left(R_{g i}+R_{g e}\right) / 2\right)\right]$.

\section{Consequence 7}

If $m \rightarrow 1$, then $r_{i}=m \cdot R_{g i} \rightarrow R_{g i}$ and $p_{i} \rightarrow+\infty$.

Comment 17. The above consequence shows that in order to obtain the inner free surface of a concave static meniscus appropriate for the growth of a tube of inner radius $r_{i} \approx R_{g i}$, $p_{i}$ has to be very high.

\section{Statement 11}

If $m$ and $p_{i}$ verify the inequalities

$$
\begin{gathered}
1<m<\frac{R_{g i}+R_{g e}}{2 \cdot R_{g i}}, \\
p_{i}>\frac{1}{m-1} \cdot \gamma \cdot \frac{\alpha_{c}+\alpha_{g}-\pi / 2}{R_{g i}} \cdot \sin \alpha_{g}+(m-1) \cdot \rho \cdot g \cdot R_{g i} \cdot \tan \alpha_{c}-\frac{\gamma}{m \cdot R_{g i}} \cdot \cos \alpha_{g}
\end{gathered}
$$

then there exist $r_{i}$ in the closed interval $\left[R_{g i}, m \cdot R_{g i}\right]$ and a concave solution of the NLBVP (2.2) and (2.5) on the interval $\left[R_{g i}, r_{i}\right]$.

Comment 18. The above statement shows that if $m$ and $p_{i}$ verify (2.21), then a static meniscus having concave inner free surface and appropriate for the growth of a tube of inner radius $r_{i}$ in the range $\left(R_{g i}, m \cdot R_{g i}\right)$, is obtained. 


\section{Consequence 8}

If for $1<m^{\prime}<m<\left(R_{g i}+R_{g e}\right) / 2 \cdot R_{g i}$ and $p_{i}$ the following inequalities hold:

$$
\begin{gathered}
\frac{1}{m-1} \cdot \gamma \cdot \frac{\alpha_{c}+\alpha_{g}-\pi / 2}{R_{g i}} \cdot \sin \alpha_{g}+(m-1) \cdot \rho \cdot g \cdot R_{g i} \cdot \tan \alpha_{c}-\frac{\gamma}{m \cdot R_{g i}} \cdot \cos \alpha_{g} \\
\quad<p_{i}<\frac{1}{m^{\prime}-1} \cdot \gamma \cdot \frac{\alpha_{c}+\alpha_{g}-\pi / 2}{R_{g i}} \cdot \cos \alpha_{c}+\frac{\gamma}{R_{g i}} \cdot \sin \alpha_{c}
\end{gathered}
$$

then there exist $r_{i}$ in the interval $\left[m^{\prime} \cdot R_{g i}, m \cdot R_{g i}\right]$ and a concave solution of the NLBVP (2.2) and (2.5) on the interval $\left[R_{g i}, r_{i}\right]$.

Comment 19. The above consequence shows that if $p_{i}$ is in the range defined by the inequalities (2.22), then a static meniscus having a concave inner free surface and appropriate for the growth of a tube of inner radius $r_{i}$, situated in the range $\left[m^{\prime} \cdot R_{g i}, m \cdot R_{g i}\right]$, is obtained.

\section{Statement 12}

A concave solution $z_{i}=z_{i}(r)$ of the NLBVP (2.2) and (2.5) is a weak minimum of the energy functional of the melt column.

Comment 20. The above statement shows that a static meniscus having a concave inner free surface appropriate for the growth of a tube of inner radius $r_{i}$, situated in the range $\left[R_{g i},\left(R_{g i}+\right.\right.$ $\left.\left.R_{g e}\right) / 2\right]$, is stable.

\section{Statement 13}

If $p_{i}$ satisfies $p_{i}<\left(-\gamma / R_{g i}\right) \cdot \sin \alpha_{c}$ and there exists $r_{i} \in\left(R_{g i}\left(R_{g i}+R_{g e}\right) / 2\right)$ such that on the interval $\left[R_{g i}, r_{i}\right]$ an appropriate static meniscus exists, then $p_{i}$ verifies:

$$
-\frac{\gamma}{R_{g i}}<p_{i}<-\frac{\gamma}{R_{g i}} \cdot \sin \alpha_{c}
$$

Comment 21. Inequality (2.23) locates the values of $p_{i}$ for which eventually nonglobally concave (convex-concave) static meniscus can be obtained.

\section{Statement 14}

If $\left(-\gamma / R_{g i}\right) \cdot \sin \alpha_{c}<p_{i}$ and there exists $r_{i}=m \cdot R_{g i} ; m \in\left(1,\left(R_{g i}+R_{g e}\right) / 2 \cdot R_{g i}\right)$ such that on $\left[R_{g i}, r_{i}\right]$ an appropriate non-globally concave static meniscus exists, then $p_{i}$ satisfies the inequalities

$$
-\frac{\gamma}{R_{g i}} \cdot \sin \alpha_{c}<p_{i}<\rho \cdot g \cdot(m-1) \cdot R_{g i} \cdot \tan \alpha_{c}+\frac{\gamma}{R_{g i}}
$$

Comment 22. Inequality (2.24) locates the values of $p_{i}$ for which eventually concave-convex static menisci can be obtained. 


\section{Statement 15}

If $m$ and $p_{i}$ satisfy the inequalities

$$
1<m<\frac{R_{g i}+R_{g e}}{2 \cdot R_{g i}}, \quad p_{i}>\frac{\gamma}{R_{g i}}+\rho \cdot g \cdot(m-1) \cdot R_{g i} \cdot \tan \alpha_{c}
$$

then the solution $z_{i}(r)$ of the IVP (2.16) is concave on the interval $I \cap\left[R_{g i}, m \cdot R_{g i}\right]$ where $I$ is the maximal interval of the existence of $z_{i}(r)$.

Comment 23. Inequality (2.25) locates the values of $p_{i}$ for which we can obtain eventually appropriate static menisci.

\section{Creation of an Appropriate Drop-Like Static Meniscus for the Growth of a Tube of Inner Radius $r_{i}$ and Outer Radius $r_{e}$}

In this section it will be shown in which kind the explicit formulas presented in the above section can be used for the creation of an appropriate drop-like meniscus when $\alpha_{c}, \alpha_{g}, \rho, \gamma, R_{g i}, R_{g e}, r_{i}$ and $r_{e}$ are given a priori. In the same time the melt column "height" $H$, which has to be used, is found in function of the pressure of the gas flow introduced in the furnace for release the heat.

\subsection{Creation of the Outer Free Surface}

For the creation of the appropriate outer free surface, the following limits were considered:

$$
\begin{aligned}
L_{1}^{e}(n) & =\frac{n}{n-1} \cdot \gamma \cdot \frac{\alpha_{c}+\alpha_{g}-\pi / 2}{R_{g e}} \cdot \cos \alpha_{c}+\frac{\gamma}{R_{g e}} \cdot \cos \alpha_{g \prime} \\
L_{2}^{e}(n) & =\frac{n}{n-1} \cdot \gamma \cdot \frac{\alpha_{c}+\alpha_{g}-\pi / 2}{R_{g e}} \cdot \sin \alpha_{g}+\frac{n-1}{n} \cdot \rho \cdot g \cdot R_{g e} \cdot \tan \alpha_{c}+n \cdot \frac{\gamma}{R_{g e}} \cdot \sin \alpha_{c}, \\
L_{3}^{e}(n) & =\frac{n-1}{n} \cdot \rho \cdot g \cdot R_{g e} \cdot \tan \alpha_{c}+n \cdot \frac{\gamma}{R_{g e}} \cdot \cos \alpha_{c}, \\
L_{4}^{e}(n) & =\frac{n-1}{n} \cdot \rho \cdot g \cdot R_{g e} \cdot \tan \alpha_{c}+n \cdot \frac{\gamma}{R_{g e}}, \\
l_{1}^{e} & =2 \cdot \gamma \cdot \frac{\alpha_{c}+\alpha_{g}-\pi / 2}{R_{g e}-R_{g i}} \cdot \cos \alpha_{c}+\frac{\gamma}{R_{g e}} \cdot \cos \alpha_{g}, \\
l_{2}^{e} & =2 \cdot \gamma \cdot \frac{\alpha_{c}+\alpha_{g}-\pi / 2}{R_{g e}-R_{g i}} \cdot \sin \alpha_{g}+\frac{\rho \cdot g \cdot\left(R_{g e}-R_{g i}\right)}{2} \cdot \tan \alpha_{c}+\frac{2 \cdot \gamma}{R_{g e}-R_{g i}} \cdot \sin \alpha_{c}, \\
l_{3}^{e} & =\frac{\gamma}{R_{g e}} \cdot \sin \alpha_{c}
\end{aligned}
$$

for $n>1$. 


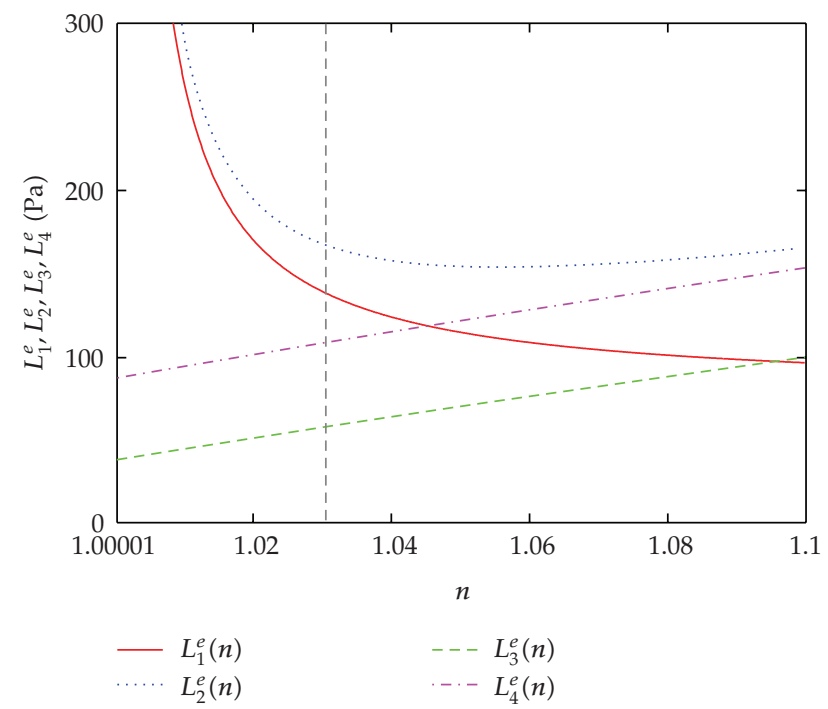

Figure 2: The possible $p_{e}$ ranges which have to be investigated for $n \in(1 ; 1.1)$.

These limits are represented for $n \in(1 ; 1.1)$ in Figure 2. The computations were performed in MathCAD V.13 using the following numerical data

$$
\begin{gathered}
R_{g e}=4.8 \cdot 10^{-3}[\mathrm{~m}] ; \quad R_{g i}=4.2 \cdot 10^{-3}[\mathrm{~m}] ; \quad \alpha_{c}=63.8^{0}=1.1135[\mathrm{rad}] \\
\alpha_{g}=28.9^{0}=0.5044[\mathrm{rad}] ; \quad \rho=6582\left[\mathrm{~kg} / \mathrm{m}^{3}\right] ; \\
\gamma=4.2 \cdot 10^{-1}[\mathrm{~N} / \mathrm{m}] ; \quad g=9.81\left[\mathrm{~m} / \mathrm{s}^{2}\right] .
\end{gathered}
$$

When $n_{1}=1.03226$, that is, $r_{e}^{1}=4.65 \cdot 10^{-3}[\mathrm{~m}]$, we have the following.

(i) If there exists a concave outer free surface, appropriate for the growth of a tube of outer radius $r_{e}^{1}=4.65 \cdot 10^{-3}[\mathrm{~m}]$, then according to Statement 3 this can be obtained for a value of $p_{e}$ which is in the range $\left(L_{1}^{e}(n), L_{2}^{e}(n)\right)=(134.85 ; 164.49)[\mathrm{Pa}]$.

(ii) Taking into account the above fact, in order to create a concave outer free surface, appropriate for the growth of a tube of which outer radius is equal to $r_{e}^{1}=$ $4.65 \cdot 10^{-3}[\mathrm{~m}]$, we have solved the IVP (2.8) for different values of $p_{e}$ in the range $(134.85 ; 164.49)[\mathrm{Pa}]$.

More precisely, we have integrated the following system:

$$
\begin{aligned}
& \frac{d z_{e}}{d r}=-\tan \alpha_{e} \\
& \frac{d \alpha_{e}}{d r}=-\frac{1}{\cos \alpha_{e}} \cdot\left[\frac{g \cdot \rho \cdot z_{e}-p_{e}}{r}+\frac{1}{r} \cdot \sin \alpha_{e}\right]
\end{aligned}
$$




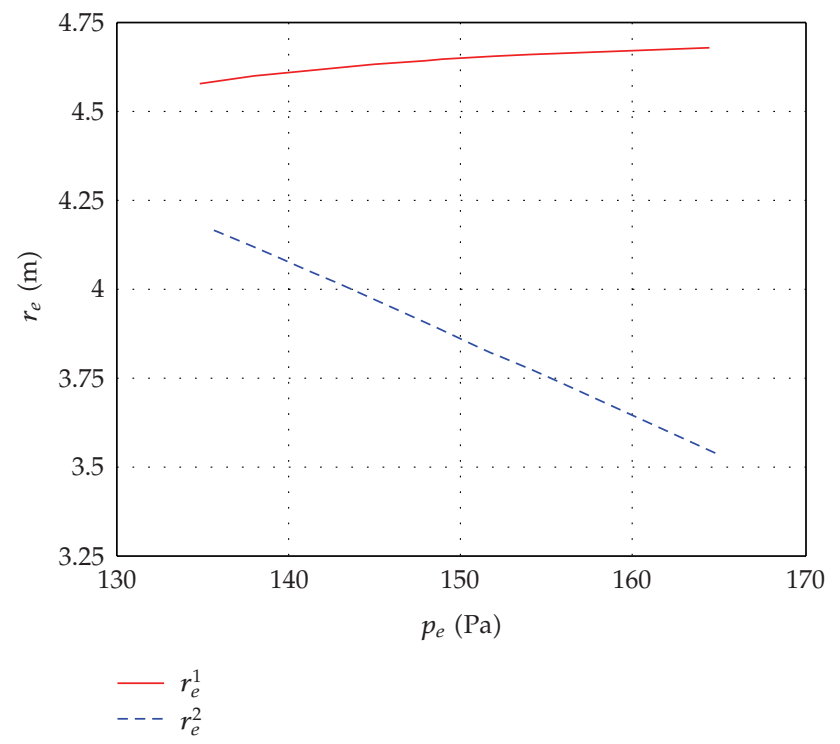

Figure 3: Outer radii $r_{e}$ versus $p_{e}$ in the range $(134.85 ; 164.49)[\mathrm{Pa}]$.

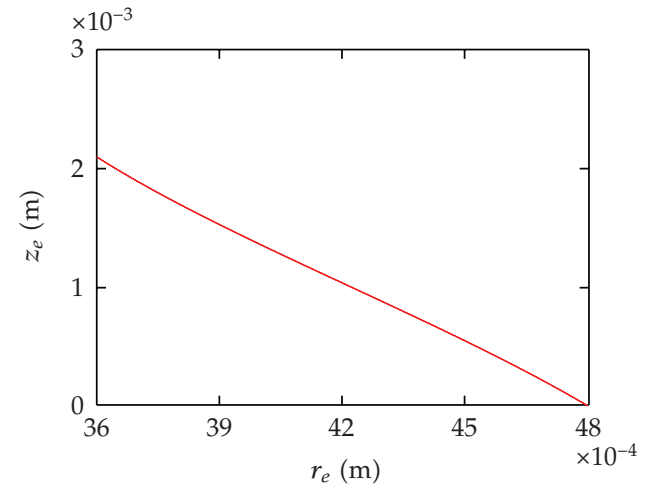

(a)

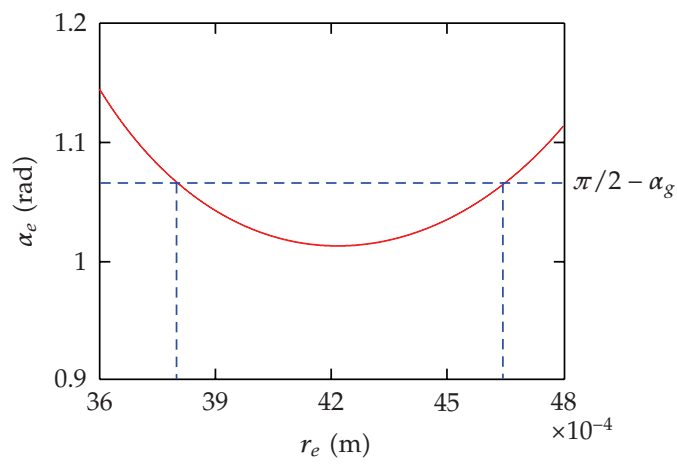

(b)

Figure 4: Nonglobally concave outer free surface obtained for $p_{e}^{\prime}=149.7[\mathrm{~Pa}]$.

for $z_{e}\left(R_{g e}\right)=0, z_{e}^{\prime}\left(R_{g e}\right)=-\tan \alpha_{c}$ and different $p_{e}$. The obtained outer radii $r_{e}$ versus $p_{e}$ are represented in Figure 3, which shows that the desired outer radius $r_{e}^{1}=4.65 \cdot 10^{-3}[\mathrm{~m}]$ is obtained for $p_{e}^{\prime}=149.7[\mathrm{~Pa}]$.

Actually, as it can be seen on the same figure, for $p_{e}^{\prime}=149.7[\mathrm{~Pa}]$, we can obtain also a second outer radius $r_{e}^{2}=3.8 \cdot 10^{-3}[\mathrm{~m}]$, which is not anymore in the desired range $\left(\left(R_{g i}+\right.\right.$ $\left.\left.R_{g e}\right) / 2, R_{g e}\right)$. Moreover, the outer free surface of this meniscus is not globally concave; it is a convex-concave meniscus (Figure 4).

Taking into account $p_{m} \approx 0[7,23,24]$, the melt column height in this case is $H_{e}^{\prime}=$ $-[1 / \rho \cdot g] \cdot\left[p_{e}^{\prime}+p_{g}^{e}\right]$, where $p_{g}^{e} \geq 0$ is the pressure of the gas flow (introduced in the furnace for release the heat from the outer side of the tube wall). When $p_{g}^{e}=0[7,24]$, then $H_{e}^{\prime}$ is negative, $H_{e}^{\prime}=-2.31 \cdot 10^{-3}[\mathrm{~m}]$; that is, the crucible melt level has to be with $-H_{e}^{\prime}=2.31 \cdot 10^{-3}[\mathrm{~m}]$ above 
the shaper top level. When $p_{g}^{e}=800[\mathrm{~Pa}]$, then $H_{e}^{\prime}=-15 \cdot 10^{-3}[\mathrm{~m}]$; that is, the crucible melt level has to be with $-H_{e}^{\prime}=15 \cdot 10^{-3}[\mathrm{~m}]$ above the shaper top level.

Additional remarks are as follows.

(i) For $p_{e}$ in the range $\left(-\infty, l_{3}^{e}\right)=(-\infty, 78.51)[\mathrm{Pa}]$, according to Statement 2 , the outer free surface of the meniscus is globally convex, and, according to Statement 1 , such a meniscus is not appropriate for the growth of a tube of outer radius $r_{e}^{1}=4.65$. $10^{-3}[\mathrm{~m}]$.

(ii) For $p_{e}$ in the range $\left(l_{3}^{e}, l_{1}^{e}\right)=(78.51 ; 105.73)[\mathrm{Pa}]$, according to Consequence 2 , it is impossible to obtain concave outer free surface, which is appropriate for the growth of a tube of outer radius $r_{e}^{1}=4.65 \cdot 10^{-3}[\mathrm{~m}]$.

(iii) For $p_{e}$ in the range $\left(l_{1}^{e}, L_{1}^{e}(n)\right)=(105.73 ; 134.85)[\mathrm{Pa}]$, according to the Statement 3 , it is impossible to obtain concave outer free surface, which is appropriate for the growth of a tube of outer radius $r_{e}^{1}=4.65 \cdot 10^{-3}[\mathrm{~m}]$.

(iv) For $p_{e}$ in the range $\left(L_{2}^{e}(n) ;+\infty\right)=(164.49 ;+\infty)[\mathrm{Pa}]$, according to the Statement 4 , the outer free surface is concave and is appropriate for the growth of a tube which outer radius $r_{e}$ is higher than $r_{e}^{1}=4.65 \cdot 10^{-3}[\mathrm{~m}]$.

\subsection{Creation of an Appropriate Inner Free Surface}

For the creation of the appropriate inner free surface, the following limits were considered

$$
\begin{aligned}
L_{1}^{i}(m) & =\frac{1}{m-1} \cdot \gamma \cdot \frac{\alpha_{c}+\alpha_{g}-\pi / 2}{R_{g i}} \cdot \cos \alpha_{c}-\frac{\gamma}{R_{g i}} \cdot \sin \alpha_{c}, \\
L_{2}^{i}(m) & =\frac{1}{m-1} \cdot \gamma \cdot \frac{\alpha_{c}+\alpha_{g}-\pi / 2}{R_{g i}} \cdot \sin \alpha_{g}+(m-1) \cdot \rho \cdot g \cdot R_{g i} \cdot \tan \alpha_{c}-\frac{1}{m} \cdot \frac{\gamma}{R_{g i}} \cdot \cos \alpha_{g}, \\
L_{3}^{i}(m) & =\rho \cdot g \cdot(m-1) \cdot R_{g i} \cdot \tan \alpha_{c}+\frac{\gamma}{R_{g i}}, \\
l_{1}^{i} & =2 \cdot \gamma \cdot \frac{\alpha_{c}+\alpha_{g}-\pi / 2}{R_{g e}-R_{g i}} \cdot \cos \alpha_{c}-\frac{\gamma}{R_{g i}} \cdot \sin \alpha_{c}, \\
l_{2}^{i} & =2 \cdot \gamma \cdot \frac{\alpha_{c}+\alpha_{g}-\pi / 2}{R_{g e}-R_{g i}} \cdot \sin \alpha_{g}+\frac{\rho \cdot g \cdot\left(R_{g e}-R_{g i}\right)}{2} \cdot \tan \alpha_{c}-\frac{2 \cdot \gamma}{R_{g e}+R_{g i}} \cdot \cos \alpha_{g}, \\
l_{3}^{i} & =-\frac{\gamma}{R_{g i}} \cdot \sin \alpha_{c}, \\
l_{4}^{i} & =-\frac{\gamma}{R_{g i}},
\end{aligned}
$$

for $m>1$.

These limits are represented for $m \in(1 ; 1.1)$ in Figure 5. The computations were performed in MathCAD V.13 using the same numerical data as for the creation of the appropriate outer free surface. 


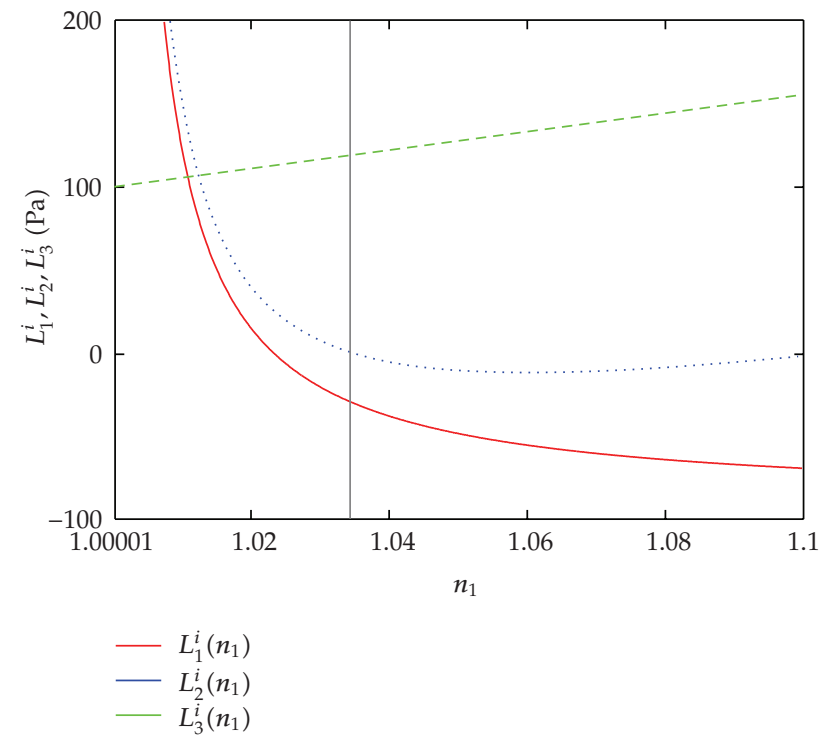

Figure 5: The possible $p_{i}$ ranges which have to be investigated for $m \in(1 ; 1.1)$.

When $m_{1}=1.03571$, that is, $r_{i}^{\prime}=0.00435[\mathrm{~m}]$, we obtain the following.

(i) If there exists a concave inner free surface for the growth of a tube of inner radius $r_{i}^{\prime}=0.00435[\mathrm{~m}]$, then, according to the Statement 10, this can be obtained for a value of $p_{i}$ which is in the range $\left(L_{1}^{i}\left(m_{1}\right), L_{2}^{i}\left(m_{1}\right)\right)=(-31.46,-1.07)[\mathrm{Pa}]$.

Taking into account the above fact, in order to create a concave inner free surface, appropriate for the growth of a tube which inner radius is equal to $r_{i}^{\prime}=0.00435[\mathrm{~m}]$, we have solved the IVP (2.16) for different values of $p_{i}$ in the range $\left(L_{1}^{i}\left(m_{1}\right), L_{2}^{i}\left(m_{1}\right)\right)=$ $(-31.46,-1.07)[\mathrm{Pa}]$. More precisely, we have integrated the following system:

$$
\begin{aligned}
& \frac{d z_{i}}{d r}=\tan \alpha_{i} \\
& \frac{d \alpha_{i}}{d r}=\frac{1}{\cos \alpha_{i}} \cdot\left[\frac{g \cdot \rho \cdot z_{i}-p_{i}}{r}-\frac{1}{r} \cdot \sin \alpha_{i}\right]
\end{aligned}
$$

for $z_{i}\left(R_{g i}\right)=0, z_{i}^{\prime}\left(R_{g i}\right)=\tan \alpha_{c}$ and different $p_{i}$. The obtained inner radii $r_{i}$ versus $p_{i}$ are represented in Figure 6, which shows that the desired inner radius $r_{i}^{1}=4.35 \cdot 10^{-3}[\mathrm{~m}]$ is obtained for $p_{i}^{\prime}=-16.2[\mathrm{~Pa}]$.

Taking $p_{m} \approx 0[7,23,24]$, the melt column height in this case is $H_{i}^{\prime}=-(1 / \rho \cdot g) \cdot\left[p_{i}^{\prime}+p_{g}^{i}\right]$, where $p_{g}^{i} \geq 0$ is the pressure of the gas flow (introduced in the furnace for release the heat from the inner side of the tube wall). When $p_{g}^{i}=0[7,24]$, then $H_{i}^{\prime}$ is positive, $H_{i}^{\prime}=0.25$. $10^{-3}[\mathrm{~m}]$; that is, the crucible melt level has to be with $H_{i}^{\prime}=0.25 \cdot 10^{-3}[\mathrm{~m}]$ under the shaper top level. When $p_{g}^{i}=800[\mathrm{~Pa}]$, then $H_{i}^{\prime}=-12.1 \cdot 10^{-3}[\mathrm{~m}]$; that is, the crucible melt level has to be with $-H_{e}^{\prime}=12.1 \cdot 10^{-3}[\mathrm{~m}]$ above the shaper top level. 


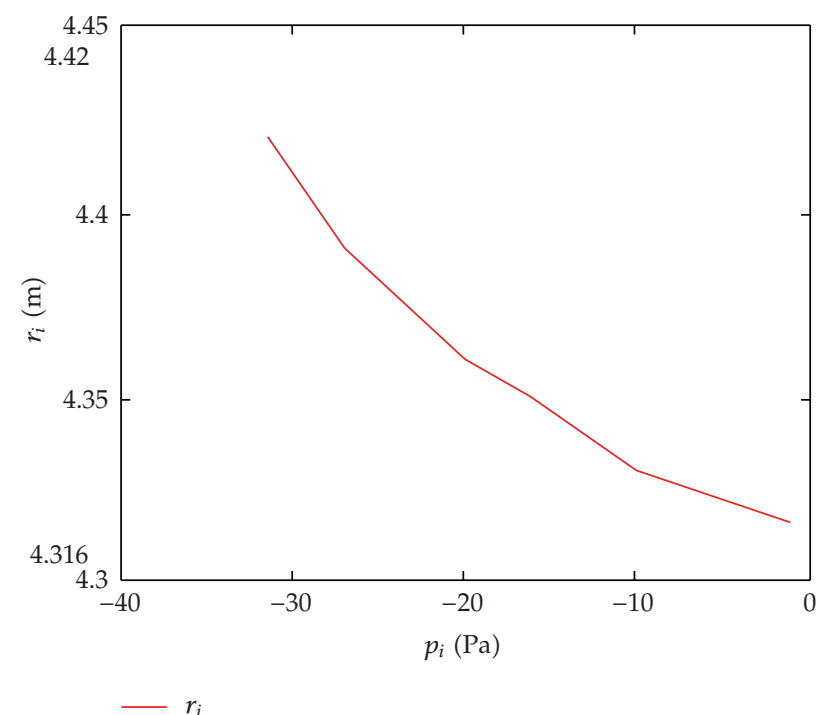

Figure 6: Inner radii $r_{i}$ versus $p_{i}$ in the range $\left(L_{1}^{i}\left(m_{1}\right), L_{2}^{i}\left(m_{1}\right)\right)=(-31.46,-1.07)[\mathrm{Pa}]$.

Additional remarks are as follows.

(i) For $p_{i}$ in the range $\left(-\infty, l_{4}^{i}\right)=(-\infty,-100)[\mathrm{Pa}]$, according to Statement 9 , the meniscus inner free surface is convex on the interval $I \cap\left[R_{g i},\left(\left(R_{g i}+R_{g e}\right) / 2\right)\right]$, and according to Statement 8 , such a meniscus is not appropriate for the growth of a tube of inner radius $r_{i}^{\prime}=0.00435[\mathrm{~m}]$.

(ii) For $p_{i}$ in the range $\left(l_{4}^{i}, l_{3}^{i}\right)=(-100,89.72)[\mathrm{Pa}]$ if an appropriate static meniscus exists, then the inner free surface of the meniscus is not globally concave.

(iii) If $p_{i}$ is in the range $\left(l_{3}^{i},+\infty\right)=(89.72,+\infty)[\mathrm{Pa}]$ and an appropriate nonglobally concave inner free surface exists, then $p_{i}$ is less than $L_{3}^{i}\left(m_{1}\right)=119.68[\mathrm{~Pa}]$.

(iv) For $p_{i}$ in the range $\left(L_{2}^{i}(m),+\infty\right)=(-1.07,+\infty)[\mathrm{Pa}]$ the obtained inner free surface is appropriate for the growth of a tube of inner radius $r_{i}$ which is less than the desired radius $r_{i}^{\prime}=0.00435[\mathrm{~m}]$.

\subsection{Creation of a Concave Meniscus, Appropriate for the Growth of a Tube of Inner Radius $r_{i}$ and Outer Radius $r_{e}$}

For creating a concave meniscus, appropriate for the growth of a tube with outer radius $r_{e}^{\prime}=$ $0.00465[\mathrm{~m}]\left(n_{1}=R_{g e} / r_{e}=1.03226\right)$ and inner radius $r_{i}^{\prime}=0.00435[\mathrm{~m}]\left(m_{1}=R_{g i} / r_{i}=1.03571\right)$ the melt column heights (with respect to the crucible melt level) have to be

$$
\begin{gathered}
H_{i}^{1}=-\frac{1}{\rho \cdot g} \cdot\left[p_{i}^{1}+p_{g}^{i}\right]=-\frac{1}{\rho \cdot g} \cdot\left[-16.2+p_{g}^{i}\right], \\
H_{e}^{1}=-\frac{1}{\rho \cdot g} \cdot\left[p_{e}^{1}+p_{g}^{e}\right]=-\frac{1}{\rho \cdot g} \cdot\left[149.7+p_{g}^{e}\right] .
\end{gathered}
$$




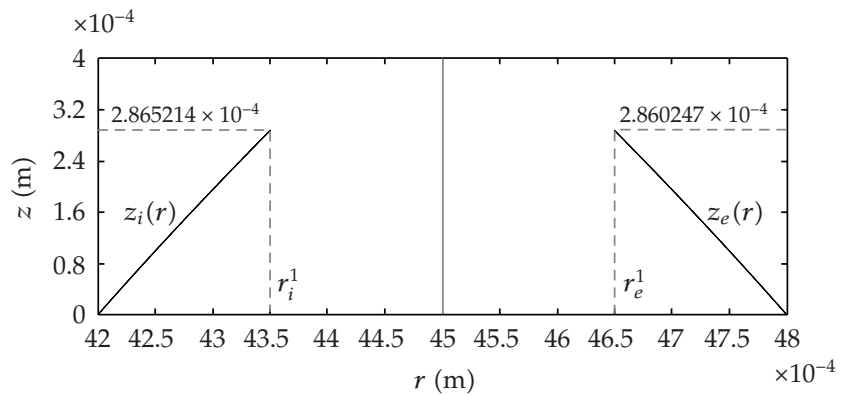

Figure 7: $z_{i}(r)$ and $z_{e}(r)$ for $p_{e}=149.7[\mathrm{~Pa}] ; p_{i}=-16.2[\mathrm{~Pa}]$ and $H_{e}^{1}=H_{i}^{1}$.

In the following we will present three different procedures to create such a meniscus.

Procedure $A\left[H_{e}^{1}=H_{i}^{1} ; p_{g}^{i} \neq p_{g}^{e}\right]$

When the shaper outer top is at the same level as the shaper inner top, with respect to the crucible melt level, then the relation $H_{e}^{1}=H_{i}^{1}$ holds and hence: $(1 / \rho \cdot g) \cdot\left[-16.2+p_{g}^{i}\right]=$ $(1 / \rho \cdot g) \cdot\left[149.7+p_{g}^{e}\right]$. It follows that the pressure of the gas flow, introduced in the furnace for releasing the heat from the inner wall of the tube, $p_{g}^{i}$, has to be higher than the pressure of the gas flow introduced in the furnace for release the heat from the outer wall of the tube, $p_{g}^{e} ; p_{g}^{i}-p_{g}^{e}=149.7+16.2=165.9[\mathrm{~Pa}]$. The results of the integration of the system (3.3) and (3.5) for $p_{e}=p_{e}^{1}=149.7[\mathrm{~Pa}]$ and $p_{i}=p_{i}^{1}=-16.2[\mathrm{~Pa}]$ when the shaper outer top is at the same level as the shaper inner top, with respect to the crucible melt level, are represented in Figure 7.Figure 7 shows also that the inner free surface is higher than the outer free surface: $z_{i}\left(r_{i}^{1}\right)=h_{i}^{a}=2.865 \cdot 10^{-4}[\mathrm{~m}] ; z_{e}\left(r_{e}^{1}\right)=h_{e}^{a}=2.860 \cdot 10^{-4}[\mathrm{~m}] ; h_{i}^{a}-h_{e}^{a}=0.005 \cdot 10^{-4}[\mathrm{~m}]$.

Procedure $B\left[p_{g}^{e}=p_{g}^{i} ; H_{e}^{1} \neq H_{i}^{1}\right]$

When the pressure of the gas flow introduced in the furnace for releasing the heat from the outer wall of the tube, $p_{g}^{e}$, is equal to the pressure of the gas flow introduced in the furnace for release the heat from the inner wall of the tube $p_{g}^{i}$, then the equalities $p_{g}^{e}=-p_{e}^{1}-\rho \cdot g \cdot H_{e}^{1}=$ $-149.7-\rho \cdot g \cdot H_{e}^{1}$ and $p_{g}^{i}=-p_{i}^{1}-\rho \cdot g \cdot H_{i}^{1}=16.2-\rho \cdot g \cdot H_{i}^{1}$ imply the equality

$$
H_{i}^{1}-H_{e}^{1}=(1 / \rho \cdot g) \cdot 165.9=2.569 \cdot 10^{-3}[\mathrm{~m}]
$$

In other words, when $p_{g}^{e}=p_{g}^{i}$, then the level difference between the shaper inner top and outer top has to be $H_{i}^{1}-H_{e}^{1}=2.569 \cdot 10^{-3}[\mathrm{~m}]$. In this condition the inner surface top level $h_{i}^{b}$ with respect to the shaper outer top level is $h_{i}^{b}=H_{i}^{1}-H_{e}^{1}+h_{i}^{a}=(1 / \rho \cdot g) \cdot\left(-p_{e}^{1}+p_{i}^{1}\right)+h_{i}^{a}=$ $2.855 \cdot 10^{-3}[\mathrm{~m}]$ (see Figure 8).

Hence the level difference between the inner free surface top $h_{i}^{b}$ and outer free surface top $h_{e}^{b}=h_{e}^{a}$ in this case is $h_{i}^{b}-h_{e}^{b}=(1 / \rho \cdot g) \cdot\left(-p_{e}^{1}+p_{i}^{1}\right)+\left(h_{i}^{a}-h_{e}^{a}\right)=2.5695 \cdot 10^{-3}[\mathrm{~m}]$.

Due to the difficulties which can appear in this case in the creation of an appropriate thermal field, which assures the solidification in the right places, it is more appropriate to use $H_{e}^{1}=H_{i}^{1}$ and gas flows having the property (Procedure A) $p_{g}^{i}=p_{g}^{e}+\left(p_{i}^{1}-p_{e}^{1}\right)=p_{g}^{e}+165.9[\mathrm{~Pa}]$. 


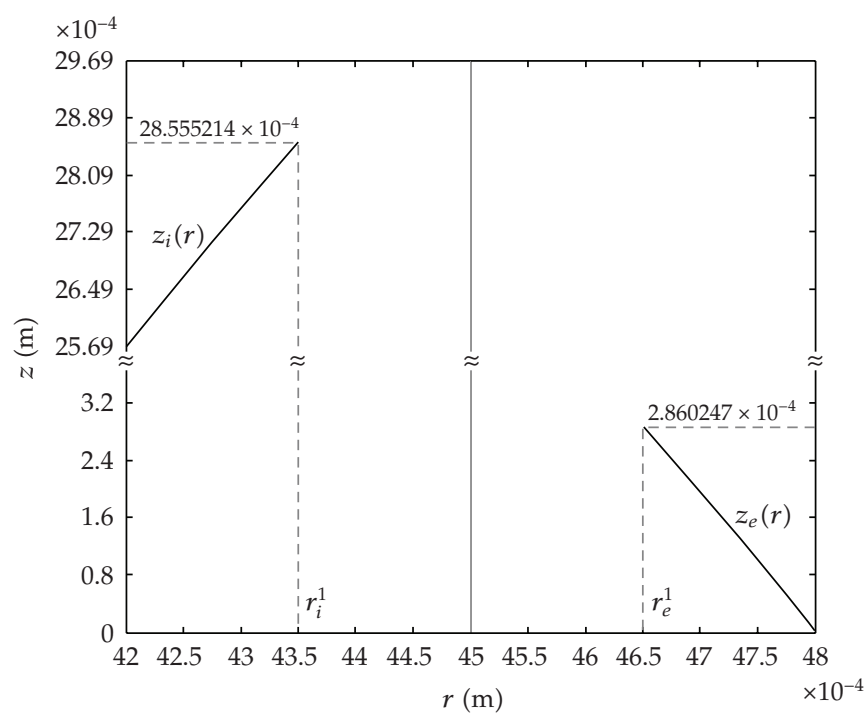

Figure 8: $z_{i}(r)$ and $z_{e}(r)$ for $p_{i}=-16.2[\mathrm{~Pa}]$ and $p_{e}=149.7[\mathrm{~Pa}]$ and $p_{g}^{e}=p_{g}^{i}$.

For this choice the difference between the inner free surface height and outer free surface height with respect to the shaper top level is only $h_{i}-h_{e}=0.0005 \cdot 10^{-3}[\mathrm{~m}]$, and the creation of the thermal field, which assures the solidification in the right places, can be easier.

\section{Procedure $C\left[p_{g}^{e} \neq p_{g}^{i} ; H_{e}^{1} \neq H_{i}^{1}\right]$}

Finally, to create an appropriate concave meniscus for which the inner meniscus is equal to the outer meniscus height, we will proceed by changing both the pressures $p_{g}^{i} p_{g}^{e}$ of the gas flows and the inner and outer top levels $H_{i}, H_{e}$ of the shaper.

For example, if we take $p_{g}^{e}=100[\mathrm{~Pa}]$ for creating an appropriate outer free surface, then the crucible melt level has to be with $-H_{e}^{1}=(1 / \rho \cdot g) \cdot[149.7+100][\mathrm{m}]=38.67 \cdot 10^{-4}[\mathrm{~m}]$ above the shaper outer top level.

Now we take $H_{i}^{1}=H_{e}^{1}-\left(h_{i}^{a}-h_{e}^{a}\right)=-38.67 \cdot 10^{-4}-0.005 \cdot 10^{-4}=-38.675 \cdot 10^{-4}[\mathrm{~m}]$. After that, in order to satisfy $p_{i}=-p_{g}^{i}-\rho \cdot g \cdot H_{i}^{1}=-16.2[\mathrm{~Pa}]$, we take the pressure $p_{g}^{i}$ equal to $p_{g}^{i}=16.2-\rho \cdot g \cdot\left(-38.675 \cdot 10^{-4}\right)=265.92[\mathrm{~Pa}]$. For this choice, we will have an appropriate drop-like meniscus for which $h_{i}^{c}=h_{e}^{c}$, as it is shown in Figure 9, obtained by integration of systems (3.3) and (3.5) for the given numerical data.

\section{The Effect of the Choice of the Shaper Radii}

In this sequence we intend to evaluate numerically the effect of the choice of the shaper radii for the growth of a tube which outer radius is equal to $r_{e}$, and inner radius is equal to $r_{i}$.

For this purpose, the procedure described in sequence 3 will be applied for the growth of a tube having the same outer radius $r_{e}^{\prime}=0.00465[\mathrm{~m}]$ and inner radius $r_{i}^{\prime}=0.00435[\mathrm{~m}]$, but the shaper radii changed: the inner radius of the shaper now is $R_{g i}=4 \cdot 10^{-3}[\mathrm{~m}]$ (instead of $4.2 \cdot 10^{-3}[\mathrm{~m}]$ ), and the outer radius of the shaper now is $R_{g e}=5 \cdot 10^{-3}[\mathrm{~m}]$ (instead of $\left.4.8 \cdot 10^{-3}[\mathrm{~m}]\right)$. 


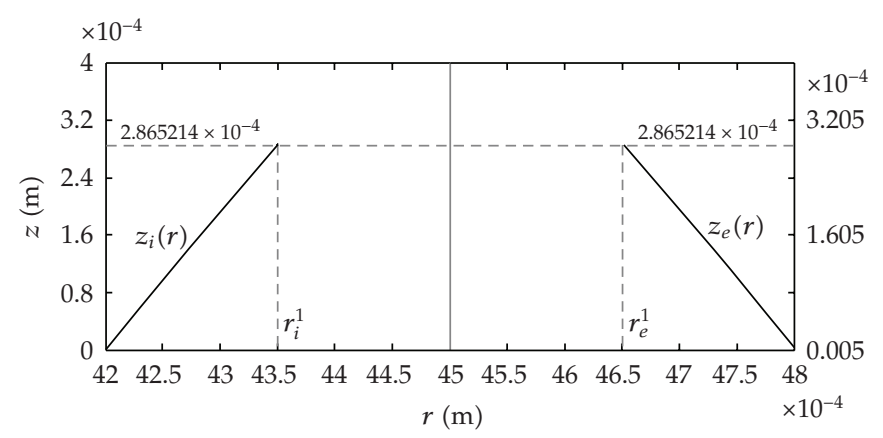

Figure 9: $z_{i}(r)$ and $z_{e}(r)$ for $p_{i}=-16.2[\mathrm{~Pa}]$ and $p_{e}=149.7[\mathrm{~Pa}] ; p_{g}^{e}=100[\mathrm{~Pa}] ; p_{g}^{i}=265.92[\mathrm{~Pa}] ; H_{e}^{1}=$ $-38.67 \cdot 10^{-4}[\mathrm{~m}] ; H_{i}^{1}=-38.675 \cdot 10^{-4}[\mathrm{~m}]$.

Applying the procedure in this case we find that to create a static meniscus which outer free surface is appropriate for the growth, $p_{e}$ has to be $p_{e}=124.38[\mathrm{~Pa}]$, and to create a static meniscus which inner free surface is appropriate for the growth, $p_{i}$ has to be $p_{i}=-41.35[\mathrm{~Pa}]$. The so-obtained outer and inner free surfaces are stable. The inner free surface height is $h_{i}=$ $6.57 \cdot 10^{-4}[\mathrm{~m}]$, and the outer free surface height is $h_{e}=6.603 \cdot 10^{-4}[\mathrm{~m}]$.

When we take the shaper inner top at the same level as the shaper outer top, then the pressure difference between the pressure of the inner gas flow $p_{g}^{i}$ and the outer gas flow $p_{g}^{e}$ has to be $p_{g}^{i}-p_{g}^{e}=p_{e}-p_{i}=165.73[\mathrm{~Pa}]$, and the level difference between the inner and outer free surface top is equal to $h_{i}-h_{e}=-0.033 \cdot 10^{-4}[\mathrm{~m}]$.

When we take the pressure of the inner gas flow $p_{g}^{i}$ equal to the pressure of the outer gas flow $p_{g}^{e}$, then the level difference between the shaper inner top $H_{i}$ and the shaper outer top $H_{e}$ has to be $H_{i}-H_{e}=(1 / \rho \cdot g) \cdot\left[p_{i}-p_{e}\right]=2.567 \cdot 10^{-3}[\mathrm{~m}]$, and the level difference between the inner and outer free surface top is equal to the difference $H_{i}+h_{i}-\left(H_{e}+h_{e}\right)=$ $(1 / \rho \cdot g) \cdot\left[p_{i}-p_{g}\right]+h_{i}-h_{e}=2.5637 \cdot 10^{-3}[\mathrm{~m}]$.

\section{Conclusions}

(i) Stable and drop-like static meniscus, appropriate for the growth of a single-crystal cylindrical tube with a priori specified inner and outer radii, can be created by the choice of the difference between the inner top level and outer top level of the shaper, or by the choice of the pressure of the gas flows, introduced inside and outside of the tube for release the heat from the tube walls.

(ii) The values of the differences between the inner and outer top level of the shaper, or the pressure in the inner and outer gas flows, depend on the shaper radii. This dependence can be relevant.

(iii) The realization of the thermal conditions, which assure that for the obtained static meniscus the solidification conditions are satisfied at the right places, is easier when we take the shaper inner top at the same level as the shaper outer top, and we use inner and outer gas flows having different pressures $\left(p_{g}^{i} \neq p_{g}^{e}\right)$. The setting of the thermal conditions is not discussed in this paper. 


\section{Acknowledgments}

The authors thank Professor Koichi Kakimoto for the useful discussions and suggestions concerning the tube growth process. They are grateful to the Romanian National Authority for Research for supporting the research under the Grant ID 354 no. 7/2007.

\section{References}

[1] T. A. Lograsso, D. L. Schlagel, and A. O. Pecharsky, "Synthesis and characterization of single crystalline $\mathrm{Gd}_{5}\left(\mathrm{Si}_{x} \mathrm{Ge}_{1-x}\right)_{4}$ by the Bridgman method," Journal of Alloys and Compounds, vol. 393, no. 1-2, pp. 141-146, 2005.

[2] C. Marín and A. G. Ostrogorsky, "Growth of Ga-doped $\mathrm{Ge}_{0.98} \mathrm{Si}_{0.02}$ by vertical Bridgman with a baffle," Journal of Crystal Growth, vol. 211, no. 1, pp. 378-383, 2000.

[3] K. Kadokura and Y. Takano, "Germanium-silicon single crystal growth using an encapsulant in a silica ampoule," Journal of Crystal Growth, vol. 171, no. 1-2, pp. 56-60, 1997.

[4] O. V. Smirnova, V. V. Kalaev, Yu. N. Makarov, N. V. Abrosimov, H. Riemann, and V. N. Kurlov, “Threedimensional unsteady modeling analysis of silicon transport in melt during $\mathrm{Cz}$ growth of $\mathrm{Ge}_{1-x} \mathrm{Si}_{x}$ bulk crystals," Journal of Crystal Growth, vol. 303, no. 1, pp. 141-145, 2007.

[5] B. Depuydt, A. Theuwis, and I. Romandic, "Germanium: from the first application of Czochralski crystal growth to large diameter dislocation-free wafers," Materials Science in Semiconductor Processing, vol. 9, no. 4-5, pp. 437-443, 2006.

[6] D. Yang, J. Chen, H. Li, et al., "Micro-defects in Ge doped Czochralski grown Si crystals," Journal of Crystal Growth, vol. 292, no. 2, pp. 266-271, 2006.

[7] L. Erris, R. W. Stormont, T. Surek, and A. S. Taylor, "The growth of silicon tubes by the EFG process," Journal of Crystal Growth, vol. 50, pp. 200-211, 1980.

[8] J. C. Swartz, T. Surek, and B. Chalmerst, "The EFG process applied to the growth of silicon ribbons," Journal of Electronic Materials, vol. 4, no. 2, pp. 255-279, 1975.

[9] T. Surek, B. Chalmers, and A. I. Mlavsky, "The edge-defined film-fed growth of controlled shape crystals," Journal of Crystal Growth, vol. 42, pp. 453-465, 1977.

[10] J. P. Kalejs, A. A. Menna, R. W. Stormont, and J. W. Hutchinson, "Stress in thin hollow silicon cylinders grown by the edge-defined film-fed growth technique," Journal of Crystal Growth, vol. 104, no. 1, pp. 14-19, 1990.

[11] S. Rajendran, C. C. Chao, D. P. Hill, J. P. Kalejs, and V. Overbye, "Magnetic and thermal field model of EFG system," Journal of Crystal Growth, vol. 109, no. 1-4, pp. 82-87, 1991.

[12] S. Rajendran, M. Larrousse, B. R. Bathey, and J. P. Kalejs, "Silicon carbide control in the EFG system," Journal of Crystal Growth, vol. 128, no. 1-4, pp. 338-342, 1993.

[13] S. Rajendran, K. Holmes, and A. Menna, "Three-dimensional magnetic induction model of an octagonal edge-defined film-fed growth system," Journal of Crystal Growth, vol. 137, no. 1-2, pp. 77-81, 1994.

[14] A. Roy, B. MacKintosh, J. P. Kalejs, Q.-S. Chen, H. Zhang, and V. Prasad, "Numerical model for inductively heated cylindrical silicon tube growth system," Journal of Crystal Growth, vol. 211, no. 1, pp. 365-371, 2000.

[15] A. Roy, H. Zhang, V. Prasad, B. Mackintosh, M. Ouellette, and J. P. Kalejs, "Growth of large diameter silicon tube by EFG technique: modeling and experiment," Journal of Crystal Growth, vol. 230, no. 1-2, pp. 224-231, 2001.

[16] D. Sun, C. Wang, H. Zhang, B. Mackintosh, D. Yates, and J. Kalejs, "A multi-block method and multigrid technique for large diameter EFG silicon tube growth," Journal of Crystal Growth, vol. 266, no. 1-3, pp. 167-174, 2004.

[17] H. Behnken, A. Seidl, and D. Franke, "A 3D dynamic stress model for the growth of hollow silicon polygons," Journal of Crystal Growth, vol. 275, no. 1-2, pp. e375-e380, 2005.

[18] B. MacKintosh, A. Seidl, M. Ouellette, B. Bathey, D. Yates, and J. Kalejs, "Large silicon crystal hollowtube growth by the edge-defined film-fed growth (EFG) method," Journal of Crystal Growth, vol. 287, no. 2, pp. 428-432, 2006.

[19] H. Kasjanow, A. Nikanorov, B. Nacke, H. Behnken, D. Franke, and A. Seidl, "3D coupled electromagnetic and thermal modelling of EFG silicon tube growth," Journal of Crystal Growth, vol. 303, no. 1, pp. 175-179, 2007. 
[20] V. A. Tatarchenko, Shaped Crystal Growth, Kluwer Academic Publishers, Dordrecht, The Netherlands, 1993.

[21] A. V. Borodin, V. A. Borodin, V. V. Sidorov, and I. S. Pet'kov, "Influence of growth process parameters on weight sensor readings in the Stepanov (EFG) technique," Journal of Crystal Growth, vol. 198-199, part 1, pp. 215-219, 1999.

[22] A. V. Borodin, V. A. Borodin, and A. V. Zhdanov, "Simulation of the pressure distribution in the melt for sapphire ribbon growth by the Stepanov (EFG) technique," Journal of Crystal Growth, vol. 198-199, part 1, pp. 220-226, 1999.

[23] S. N. Rossolenko, "Menisci masses and weights in Stepanov (EFG) technique: ribbon, rod, tube," Journal of Crystal Growth, vol. 231, no. 1-2, pp. 306-315, 2001.

[24] B. Yang, L. L. Zheng, B. Mackintosh, D. Yates, and J. Kalejs, "Meniscus dynamics and melt solidification in the EFG silicon tube growth process," Journal of Crystal Growth, vol. 293, no. 2, pp. 509-516, 2006.

[25] St. Balint and L. Tanasie, "Nonlinear boundary value problems for second order differential equations describing concave equilibrium capillary surfaces," Nonlinear Studies, vol. 15, no. 4, pp. 277-296, 2008. 


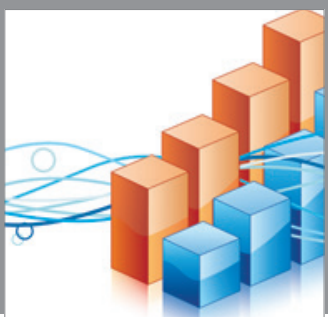

Advances in

Operations Research

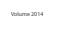

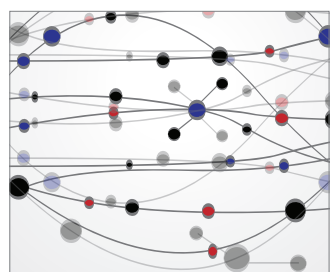

\section{The Scientific} World Journal
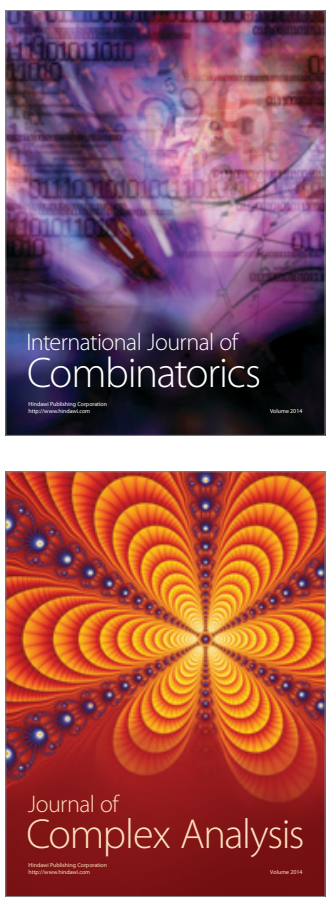

International Journal of

Mathematics and

Mathematical

Sciences
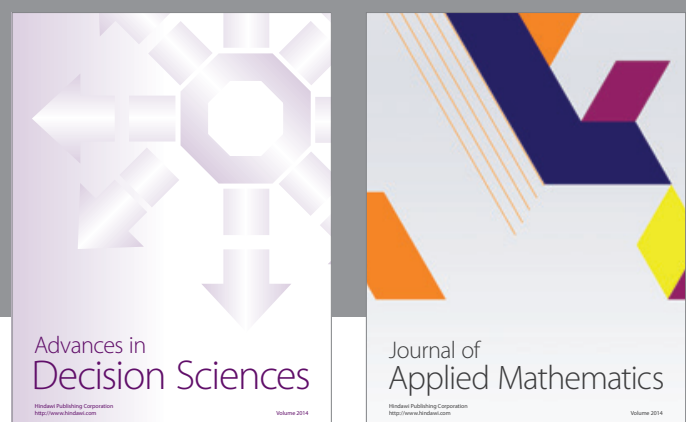

Journal of

Applied Mathematics
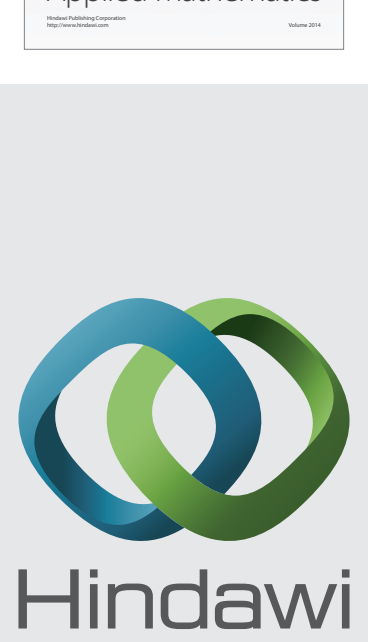

Submit your manuscripts at http://www.hindawi.com
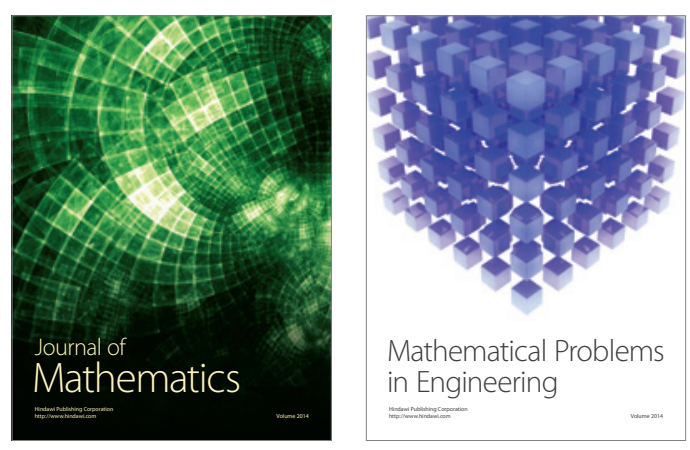

Mathematical Problems in Engineering
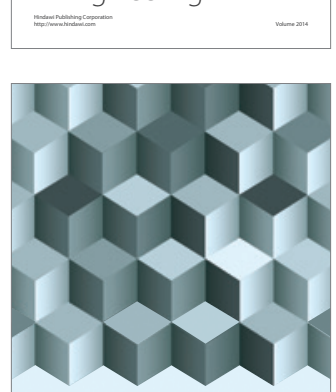

Journal of

Function Spaces
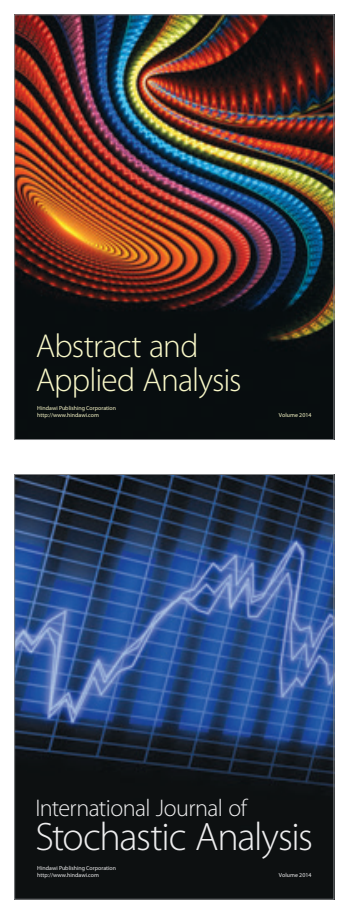

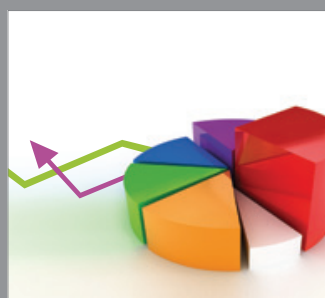

ournal of

Probability and Statistics

Promensencen
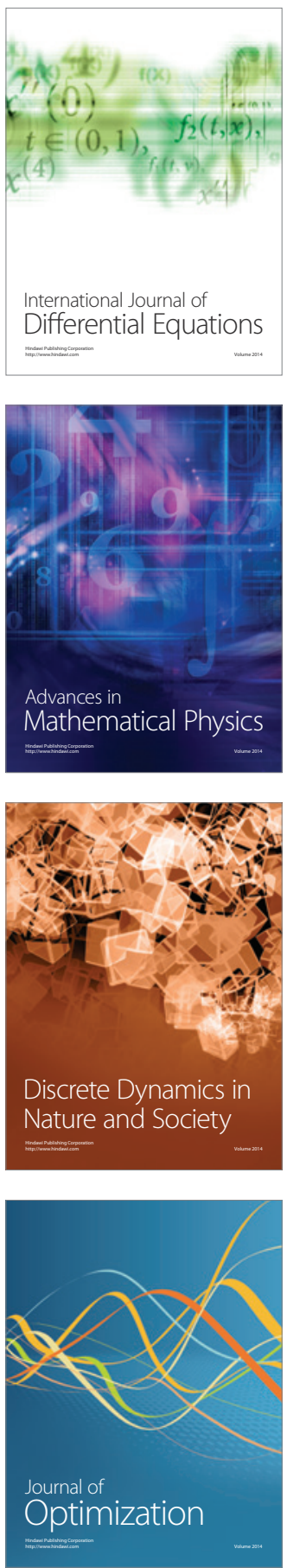